\title{
Correction to: Quantitative variation and heritability estimates of fire blight resistance in a pedigree-connected apple germplasm set
}

\author{
Sarah A. Kostick ${ }^{1} \cdot$ John L. Norelli ${ }^{2} \cdot$ Soon Li Teh ${ }^{1} \cdot$ Kate M. Evans ${ }^{1}$
}

Published online: 26 May 2020

(C) Società Italiana di Patologia Vegetale (S.I.Pa.V.) 2020

\section{Correction to: Journal of Plant Pathology https://doi.org/10.1007/s42161-020-00543-0}

The article was published with errors in Table 1 and Online Resource 1 found in the results section and supplementary material. In Table 1 and Online Resource 1, incorrect numbers of seedlings were listed for Family 9 (Cripps Pink x Honeycrisp) and Family 25 (Honeycrisp x Splendour). This error also impacted some information in Online Resource 1. The author group and publisher regret the errors and ask the following to be considered instead.
The online version of the original article can be found at https://doi.org/ 10.1007/s42161-020-00543-0

Electronic supplementary material The online version of this article (https://doi.org/10.1007/s42161-020-00571-w) contains supplementary material, which is available to authorized users.

Kate M. Evans

kate_evans@wsu.edu

1 Department of Horticulture, Tree Fruit Research and Extension Center, Washington State University, Wenatchee, WA 98801, USA

2 United States Department of Agriculture, Agricultural Research Service, Appalachian Fruit Research Laboratory, Kearneysville, WV 25430, USA
In the original article, Table 1 lists incorrect numbers of seedlings for Families 9 (Cripps Pink x Honeycrisp) and 25 (Honeycrisp x Splendour). The correct numbers of seedlings for these families should be 7 Cripps Pink x Honeycrisp seedlings and 2 Honeycrisp x Splendour seedlings. This can be found in the attached Table 1 corrected.

Additionally, information in Online Resource 1 for these families should be replaced with information provided in the attached Online Resource 1 corrected. 
Table 1 Parentage and seedling numbers of 32 pedigreeconnected $\mathrm{F}_{1}$ full-sib families

\begin{tabular}{|c|c|c|c|}
\hline \multirow[t]{2}{*}{ Family \# } & \multicolumn{2}{|l|}{ Parents ${ }^{\mathrm{z}}$} & \multirow[t]{2}{*}{ \# Seedlings } \\
\hline & Maternal & Paternal & \\
\hline 1 & Aurora Golden Gala (MR) & Arlet (MR) & 11 \\
\hline 2 & Aurora Golden Gala (MR) & Enterprise (HR) & 9 \\
\hline 3 & Aurora Golden Gala (MR) & Granny Smith (HS) & 9 \\
\hline 4 & Aurora Golden Gala (MR) & Honeycrisp (MS) & 31 \\
\hline 5 & Braeburn & Ginger Gold (HS) & 24 \\
\hline 6 & Cripps Pink (MS) & Aurora Golden Gala (MR) & 12 \\
\hline 7 & Cripps Pink (MS) & Braeburn & 2 \\
\hline 8 & Cripps Pink (MS) & Delicious (MR) & 3 \\
\hline 9 & Cripps Pink (MS) & Honeycrisp (MS) & 7 \\
\hline 10 & Cripps Pink (MS) & WA5 & 17 \\
\hline 11 & Cripps Pink (MS) & W.7 & 24 \\
\hline 12 & Delicious (MR) & Honeycrisp (MS) & 9 \\
\hline 13 & Enterprise (HR) & Arlet (MR) & 6 \\
\hline 14 & Fuji (MR) & NY06 & 5 \\
\hline 15 & Ginger Gold (HS) & Minnewashta (MS) & 2 \\
\hline 16 & Honeycrisp (MS) & Arlet (MR) & 14 \\
\hline 17 & Honeycrisp (MS) & Enterprise (HR) & 4 \\
\hline 18 & Honeycrisp (MS) & Golden Delicious (MR) & 1 \\
\hline 19 & Honeycrisp (MS) & Honeygold & 1 \\
\hline 20 & Honeycrisp (MS) & Minnewashta (MS) & 6 \\
\hline 21 & Honeycrisp (MS) & UMN Selection 1839 & 1 \\
\hline 22 & Honeycrisp (MS) & MN1964 & 19 \\
\hline 23 & Honeycrisp (MS) & Regent & 7 \\
\hline 24 & Honeycrisp (MS) & Silken & 10 \\
\hline 25 & Honeycrisp (MS) & Splendour (I) & 2 \\
\hline 26 & Minnewashta (MS) & BC8S-27-43 & 7 \\
\hline 27 & Pinova (HS) & NY03 & 22 \\
\hline 28 & Sansa (HS) & Fuji (MR) & 1 \\
\hline 29 & Sansa (HS) & Granny Smith (HS) & 11 \\
\hline 30 & Sunrise (MR) & Minnewashta (MS) & 1 \\
\hline 31 & Sweet Sixteen (HS) & BC8S-27-43 & 14 \\
\hline 32 & W.7 & WA5 & 22 \\
\hline Total & & & 314 \\
\hline
\end{tabular}

${ }^{\mathrm{z}}$ Resistance/susceptibility classifications (i.e., highly resistant [HR], moderately resistant [MR], intermediate [I], moderately susceptible [MS], highly susceptible [HS]) of parents included for those evaluated by Kostick et al. (2019) 